\title{
Serological survey for RHD antibodies in rabbits from two types of rabbit breeding farms
}

\author{
A. Fitzner, W. Niedbalski \\ Department of Foot and Mouth Disease, National Veterinary Research Institute, \\ 98-220 Zduńska Wola, Wodna 7, Poland
}

\begin{abstract}
Seroprevalence studies of RHDV antibodies in domestic rabbits were conducted between 2008-2014. A total of 12,169 sera from the provinces of central, southern and south-east Poland, including 7,570 samples collected from mixed-breed rabbits reared in smallholder farms and nearly 4,600 sera taken mainly from unvaccinated rabbits kept in industrial farms, were examined using ELISA tests. Additionally, cross-reactivity of selected tested and control archival sera using both classic RHDV and RHDVa antigens was determined by HI assay. The overall seroprevalence was 13.3\%. In rabbits with unkown history of immunisation or RHD infection which came from small farms, RHDV antibodies were detected in $6.1 \%$ ranging between $1.0 \%$ to $17.2 \%$ of animals. In rabbits of the same group, but with a declared vaccination status, or confirmed exposure to an infectious virus, or coming from exposed females, the seroprevalence ranged from $83 \%$ to $100 \%$. Among unvaccinated meat rabbits aged 71 to 90 days from industrial farms, low $(1.85 \%, 4.17 \%$, $11 \%)$, medium $(34 \%, 54 \%)$ or high rates $(98.7 \%)$ of seropositivity were detected. The seroconversion recorded in adult vaccinated females from industrial farms was $70 \%$ and $95 \%$. Generally, the antibody levels examined by ELISAs and HI were comparable. However, a number of sera from the rabbits from small farms, as well as archival sera, showed clear differences. Several-fold differences in antibody titers, evidenced mainly in the postoutbreak sera, indictaed the contact of animals with RHDVa antigen. The overall results of the survey revealed a great proportion of seronegative rabbits potentially highly susceptible to RHD infection. In combination with the emergence of a novel pathogenic RHD virus type (RHDV2), it poses a severe risk of a next wave of fatal disease cases spreading in the native population of domestic rabbits, especially in farms with a traditional system of husbandry.
\end{abstract}

Key words: RHD, serology, domestic rabbits, industrial farms, smallholder farms 


\section{Introduction}

Rabbit haemorrhagic disease (RHD), a highly contagious and fatal disease of domestic rabbits (Oryctolagus cuniculus), was first observed in China in 1984 (Liu et al. 1984). The beginning of RHD epizootic in Europe dates back to 1986 (Cancellotii and Renzi 1991). Within two years, a deadly wave of rabbit infections spread rapidly to other European countries. At the same time, RHD affected rabbits in different countries of Asia, North Africa and Mexico on the North American continent (Morisse et al. 1991). A common, characteristic feature of all RHD epizootics of this period was the susceptibility to infection of animals usually over two months old, very high morbidity and mortality rates, at times reaching $100 \%$. Equally fatal effects caused by RHD were reported in European populations of wild rabbits, leading to an imbalance of biocenosis among the predators feeding on rabbits (Marchandeau et al. 1998, Delibes-Mateos et al. 2014). The emergence of the disease in the Australian continent in the mid-90s was associated with uncontrolled escape of the RHD virus tested on Wardang island. In New Zealand the virus was introduced as a biological weapon to control the population of wild rabbits (Cooke et al. 2000). The disease is caused by RHD virus (RHDV), an RNA virus classified as a representative of the Lagovirus genus within the Caliciviridae. Identification of the etiological agent and recognition of its main morphological, physicochemical and biological properties (Ohlinger et al. 1990) led to the development of the classical virological methods (HA, immunoassay) for routine diagnosis of RHD, as well as the methods of vaccine production based on the organ homogenates of infected rabbits. The first inactivated vaccines contributed to the reduction of the number of infections and were used to control the spread of the virus in the environment. Early studies performed in the 1980s and the 1990s showed antigenic and genetic stability of RHDV (Berninger and House 1995, Le Gall et al. 1998). Recent studies have provided a more potent differentiation of pathogenic RHDV into three separate groups (classical RHDV, RHDVa, RHDV2) as well as a non-pathogenic form of the rabbit calicivirus (RCV, RCV A1) (Capucci et al. 1996, 1998, Bruce and Twigg 2004, Abrantes et al. 2012, Le Gall-Reculé et al. 2013).

The first outbreaks of RHD in Poland were confirmed in 1988 (Górski et al. 1988). At present, RHDV is still circulating in the environment, continuing to cause new epidemics, but on a much smaller scale. Two subtypes of RHDV have been identified on the basis of antigenic and genetic properties: classical RHDV (including HA-negative phenotypic vari- ant) and RHDVa, diagnosed since the middle of the first decade of the new millenium (Chrobocińska and Mizak 2007, Fitzner 2009). No infectious subtype RHDV2 or non-pathogenic RCV has been reported so far. The rabbit population in Poland is currently estimated at $15-16$ million animals. About $75-80 \%$ of these are located in small-scale farms, and the rest in commercial, industrial farms where intensive rabbit meat production is carried out. Planned vaccinations as a method of RHD control are only provided on industrial farms, where active immunization of the female breeders is implemented. Conversely, in small rural farms vaccinations are conducted irregularly. Usually both adult and young (6- to 8-week-old) rabbits are immunized. The use of vaccines in RHD prevention is estimated at $10 \%$ of the overall rabbit population.

The purpose of this study was to evaluate the current RHD serological status of domestic rabbits from two different breeding systems: 1) extensive, traditional, with open-air production, unprotected against the intrusion of infectious agents from the external environment; and 2) intensive husbandry of industrial farms. Other issues related to the search for serological evidence of RHDV circulation in the environment, with regard to a seasonal increase in the overall population of rabbits, the emergence of a large number of susceptible animals and intensification of outbreaks, mostly during the summer. The second aim of the study was comparison of the serological response in HI assay using classical RHDV and RHDVa subtype antigens, to evaluate RHDV antibody in rabbit test and archival sera collected at another time.

\section{Materials and Methods}

Sera. A total of 12,169 rabbit sera from the provinces of central, southern and south-east regions of Poland were collected between 2008-2014, in the period from early spring to late autumn (Table 1). $10 \mathrm{ml}$ blood samples were taken randomly from healthy rabbits during the slaughter. A panel of 7,570 field sera from mixed-breed rabbits reared at smallholder farms was collected during 5 years. The rabbits with RHD unknown immunological status were sampled at the age of 3 to 5 months (weighing approx. $3.5 \mathrm{~kg}$ ). Several blood samples were collected from the marginal ear vein of rabbits (series 042011) from healthy vaccinated NZW rabbits and four batches (022013a-d) from the rabbitries where RHD outbreaks were confirmed a few months earlier, directly from the small farms. More than 4,500 unvaccinated rabbits from industrial farms were sampled between 2011-2013. On these farms, in the entire lifecycle of the rabbits, last- 
Table 1. List and origin of samples.

\begin{tabular}{|c|c|c|c|c|c|c|}
\hline Batch & $\begin{array}{l}\text { Type of } \\
\text { farming } \\
\text { / breed }\end{array}$ & $\begin{array}{l}\text { Date of } \\
\text { collection }\end{array}$ & RHD vaccination status / type of vaccine & $\begin{array}{l}\text { Age of } \\
\text { rabbits }\end{array}$ & $\begin{array}{l}\text { Serum } \\
\text { samples } \\
\text { No. }\end{array}$ & $\begin{array}{l}\text { Virological } \\
\text { samples } \\
\text { (liver) No. }\end{array}$ \\
\hline 012008 & $\mathrm{~S} / \mathrm{m}-\mathrm{b}$ & Sept & Unknown & 3-8 M & 283 & 15 \\
\hline 022008 & $\mathrm{~S} / \mathrm{m}-\mathrm{b}$ & Oct & Unknown & 3-8 M & 360 & 16 \\
\hline 032008 & $\mathrm{~S} / \mathrm{m}-\mathrm{b}$ & Oct & Unknown & 3-8 M & 450 & 17 \\
\hline 042008 & $\mathrm{~S} / \mathrm{m}-\mathrm{b}$ & Nov & Unknown & 3-8 M & 450 & 15 \\
\hline 012009 & $\mathrm{~S} / \mathrm{m}-\mathrm{b}$ & Mar & Unknown & 3-8 M & 270 & 0 \\
\hline 022009 & $\mathrm{~S} / \mathrm{m}-\mathrm{b}$ & Sep & Unknown & 3-8 M & 450 & 10 \\
\hline 032009 & $\mathrm{~S} / \mathrm{m}-\mathrm{b}$ & Sep & Unknown & 3-8 M & 479 & 11 \\
\hline 042009 & $\mathrm{~S} / \mathrm{m}-\mathrm{b}$ & Nov & Unknown & 3-8 M & 500 & 12 \\
\hline 052009 & $\mathrm{~S} / \mathrm{m}-\mathrm{b}$ & Dec & Unknown & 3-8 M & 500 & 0 \\
\hline 012010 & $\mathrm{~S} / \mathrm{m}-\mathrm{b}$ & Sep & Unknown & 3-8 M & 540 & 0 \\
\hline 022010 & $\mathrm{~S} / \mathrm{m}-\mathrm{b}$ & Nov & Unknown & 3-8 M & 420 & 0 \\
\hline 012011 & $\mathrm{I}-\mathrm{F} / \mathrm{Hy}$ & Mar & unvac. offspring of vaccinated dams & $85 \mathrm{D}$ & 10 & 0 \\
\hline 022011 & I-A/ Hy & May & unvac. offspring of vaccinated dams (2xb) & $85 \mathrm{D}$ & 540 & 11 \\
\hline 032011 & $\mathrm{~S} / \mathrm{m}-\mathrm{b}$ & Jun & Unknown & 3-8 M & 420 & 10 \\
\hline 042011 & $\mathrm{~S} / \mathrm{NZW}$ & Aug & vaccinated $(1 \mathrm{xb})$ & 3-4 M & 42 & 10 \\
\hline 52011 & I-D / Hy & Sep & unvac. offspring of vaccinated dams & $85 \mathrm{D}$ & 540 & 10 \\
\hline 062011 & $\mathrm{~S} / \mathrm{m}-\mathrm{b}$ & Nov & Unknown & 3-8 M & 540 & 5 \\
\hline 072011 & $\mathrm{I}-\mathrm{B} / \mathrm{HyT}$ & Dec & unvac. offspring of vaccinated dams (1xb) & $85 \mathrm{D}$ & 540 & 8 \\
\hline 012012 & $\mathrm{I}-\mathrm{B} / \mathrm{HyT}$ & Mar & unvac. offspring of vaccinated dams $(1 \mathrm{xb})$ & $85 \mathrm{D}$ & 540 & 12 \\
\hline 022012 & $\mathrm{I}-\mathrm{B} / \mathrm{HyT}$ & Mar & unvac. offspring of vaccinated dams (1xb) & $85 \mathrm{D}$ & 480 & 11 \\
\hline 032012 & I-A/ Hy & Apr & unvac. offspring of vaccinated dams (2xb) & $85 \mathrm{D}$ & 459 & 10 \\
\hline 032012a & $\mathrm{I}-\mathrm{A} / \mathrm{Hy}$ & Apr & vaccinated dams $(2 \mathrm{xb})$ & $18-24 \mathrm{M}$ & 60 & 10 \\
\hline 042012 & $\mathrm{~S} / \mathrm{m}-\mathrm{b}$ & Sep & Unknown & 3-8 M & 540 & 10 \\
\hline 052012 & $\mathrm{~S} / \mathrm{m}-\mathrm{b}$ & Oct & Unknown & 3-8 M & 540 & 8 \\
\hline 012013 & $\mathrm{I}-\mathrm{C} / \mathrm{Hy}$ & May & unvac. offspring of vaccinated dams $(2 x)$ & $71 \mathrm{D}$ & 540 & 8 \\
\hline $02 \mathrm{a} 2013$ & $\mathrm{~S} / \mathrm{m}-\mathrm{b}$ & May & $\begin{array}{c}\text { vaccinated dams, } 4 \mathrm{M} \text { after RHD outbreak } \\
\text { unvac. offspring of vaccinated dams, } 4 \mathrm{M} \text { after } \\
\text { RHD outbreak }\end{array}$ & $\begin{array}{c}12 \mathrm{M} \\
5 \mathrm{M}\end{array}$ & $\begin{array}{l}2 \\
8\end{array}$ & 0 \\
\hline $02 \mathrm{~b} 2013$ & $\mathrm{~S} / \mathrm{m}-\mathrm{b}$ & May & $\begin{array}{l}\text { vaccinated dams, } 4 \mathrm{M} \text { after RHD outbreak } \\
\text { unvac. offspring of vaccinated dams }\end{array}$ & $\begin{array}{c}8-12 \mathrm{M} \\
6 \mathrm{~W}\end{array}$ & $\begin{array}{l}4 \\
2\end{array}$ & 0 \\
\hline $02 \mathrm{c} 2013$ & $\mathrm{~S} / \mathrm{Rex}$ & May & $\begin{array}{c}\text { vaccinated (3 dams, } 1 \text { buck) } \\
4 \mathrm{M} \text { after RHD outbreak } \\
\text { vaccinated offspring of vaccinated dams }\end{array}$ & $\begin{array}{l}12-36 \mathrm{M} \\
3-4 \mathrm{M}\end{array}$ & $\begin{array}{l}5 \\
4\end{array}$ & 1 \\
\hline $02 \mathrm{~d} 2013$ & $\mathrm{~S} / \mathrm{m}-\mathrm{b}$ & May & $\begin{array}{c}\text { vaccinated dams/ } 4 \mathrm{M} \text { after RHD outbreak } \\
\text { vaccinated offspring of doe no. } 1 \\
\text { unvaccinated offspring of doe no. } 2\end{array}$ & $\begin{array}{c}24-36 \mathrm{M} \\
3 \mathrm{M} \\
6 \mathrm{~W}\end{array}$ & $\begin{array}{l}2 \\
4 \\
5\end{array}$ & 0 \\
\hline 032013 & $\mathrm{I}-\mathrm{C} / \mathrm{Hy}$ & Jul & unvac. offspring of vaccinated dams (2xb) & $71 \mathrm{D}$ & 540 & 10 \\
\hline 042013 & $\mathrm{~S} / \mathrm{m}-\mathrm{b}$ & Jun & Unknown & 3-8 M & 540 & 12 \\
\hline 052013 & I-E / T & Nov & unvac. offspring of vaccinated dams $(2 x)$ & $90 \mathrm{D}$ & 330 & 10 \\
\hline 062013 & $\mathrm{~S} / \mathrm{m}-\mathrm{b}$ & Nov & Unknown & 3-8 M & 210 & 0 \\
\hline 012014 & $\mathrm{I}-\mathrm{G} / \mathrm{Hy}$ & Dec & vaccinated dams $(2 \mathrm{xb})$ & $12-18 \mathrm{M}$ & 20 & 4 \\
\hline
\end{tabular}

S - small-scale farm, I - industrial farm (A, B, C, D, E, F, G); Breed: NZW - New Zealand white, T- Termond white , R - Rex, Hy - hybrid Hyplus, m-b - mixed-breed; D - days; W - week, M - months; unvacc. - unvaccinated; b - bivalent vaccine (RHD-myxomatosis); $1 \mathrm{x} / 2 \mathrm{x}$ - number of vaccinations 
ing about 1.5 years, the does are mostly vaccinated twice, using mono or bivalent (for RHD and Myxomatosis) commercial vaccines. The young rabbits are not vaccinated during the fattening period, which lasts up to $11-13$ weeks. The rabbits were slaughtered after reaching a weight of $2.5-2.6 \mathrm{~kg}$ at the age of $71-90$ days. In one confirmed case, blood samples originated from 60 does (aged 18-24 months) vaccinated against RHD, from liquidated maternal livestock (batch 032012a). On farms A, C and E at least double immunization of the female cohort was declared. On farm $\mathrm{B}$, a single vaccination schedule of the dams was implemented. The strategy and the number of immunizations on farms $\mathrm{D}$ and $\mathrm{F}$ has not been established. Additionally, a small batch of sera intended for laboratory analysis in 2014 were taken from industrial farm rabbits declared as vaccinated against RHD. All sera were stored frozen at below $-18^{\circ} \mathrm{C}$ until used.

Archival sera (RHD positive reference). For a comparative study, three sets of archival RHDV positive rabbit sera (post-vaccination, post-vaccination and infection, postoutbreak) from 1994, 2004 and of 2009 were included. Postvaccinal serum samples included 160 sera from the 3, 6, 9 and 12-month-old New Zealand White (NZW) rabbit females vaccinated against RHD on an industrial farm (four groups, 40 samples each, collected at the end of December 2004). The subset consisted of 40 sera from 10 representatives of each tested group, collected 14 days after challenge at the laboratory facility. In turn, postvaccination and infection sera consisted of 15 serum samples from NZW rabbits immunised with experimental vaccines consisting of inactivated RHDVa or RHDV HA-negative, collected 2 weeks after vaccination, and subsequently 2 weeks after challenge with homologous and heterologous RHDV, under laboratory conditions (Fitzner 2009). Finally, postoutbreak sera included 22 samples of sera from unvaccinated NZW rabbits collected 18 days after the beginning of an RHD epizootic caused by classical RHDV (strain MAL) on an industrial farm in 1994 (specimens treated as coming from rabbit survivors of RHDV) and 10 serum samples from Flemish (Belgian) Giant rabbits, declared as vaccinated against RHD with monovalent vaccine, collected 11 days after the beginning of an RHD outbreak caused by classic RHDV (HA negative strain OPO) in 2004 (Fitzner 2009).

Liver. The specimens from healthy rabbits were used for RHDV antigen studies. A total of 247 liver tissue samples were collected randomly from slaughtered animals. 143 livers originated from mixed breed rabbits and 104 from meat rabbits.

Serological methods. All rabbit sera were examined for RHD antibodies by liquid-phase blocking ELISA (LPBE) (Fitzner and Niedbalski 1996). Based on the point result at a dilution of 1:10, positive sera were titrated from 1:10 to 1:20,480 (two-fold dilution). The titre of antibodies was expressed as the reciprocal final dilution of serum equal to $50 \%$ of the OD490 nm of control antigen. Simultaneously, for comparison, a portion of positive serum samples were tested using commercial cELISA with monoclonal antibodies (Mab) according to the manufacturer's instructions (IZSLER) and by the haemagglutination inhibition method (HI). The haemagglutination inhibition (HI). Test was carried out on a U-bottomed microtitration plate. Before testing, the sera were inactivated at $56^{\circ} \mathrm{C}$ for $30 \mathrm{~min}$ and treated with a $25 \%$ solution of caolin at $25^{\circ} \mathrm{C}$ for $20 \mathrm{~min}$. Serial two-fold dilutions of serum samples, starting from 1:10, were performed in a volume of $50 \mu \mathrm{l}$ in $0.85 \%$ phosphate buffered saline (PBS) ( $\mathrm{pH} 7.5)$, mixed with an equal volume of viral antigen for $30 \mathrm{~min}$ at $18-26^{\circ} \mathrm{C}$, and incubated with 100 $\mu \mathrm{l}$ of $0.75 \%$ human red blood cell suspension (type "0") at $18-26^{\circ} \mathrm{C}$ for $1.5 \mathrm{~h}$. The antibody level was measured against 4 HA units of classic RHDV (KGM), subtype RHDVa (GRZ strain) and expressed as the reciprocal of serum dilutions with full inhibition of haemagglutination.

Virological examinations. To detect RHDV antigen, $20 \% \mathrm{w} / \mathrm{v}$ of rabbit liver homogenates were examined by haemagglutination test (HA) with human „0" red blood cells and two ELISAs. The haemagglutination was assayed in $0.85 \% \mathrm{NaCl}(\mathrm{pH} \mathrm{7.5)}$ at room temperature $\left(18-26^{\circ} \mathrm{C}\right)$, and in $\mathrm{PBS}(\mathrm{pH} \mathrm{6.5)}$ at $+5^{\circ} \mathrm{C}\left( \pm 3^{\circ} \mathrm{C}\right)$. The results were read visually after 1.5-2 $\mathrm{h}$ of incubation. A sandwich ELISA kit composed of chicken and guinea pig polyclonal antisera against classical RHDV (Fitzner and Niedbalski 1996), and a commercial Mab ELISA kit (IZSLER) containing specific polyclonal antiserum (capture antibodies) and monoclonal 1H8 antibodies (detecting antibodies) were used. In both ELISAs the samples were tested at dilutions of 1:5 and 1:30, and the $\mathrm{OD}_{490} \mathrm{~nm}$ were compared with positive and negative control antigens.

\section{Results}

Serology. Test sera. Out of the total number of tested sera, 1,621 (13.3\%) were found RHD positive in LPBE and confirmed by cELISA. The average percentage of positive results in animals from the individual types of breeding was highly diversified and stood at about $25 \%$ in industrial farms, compared to $6 \%$ in smallholder farms. The detailed results of the serological examination for RHDV antibodies in mixed-breed rabbits from small-scale farms and in meat rabbits from industrial farms are presented in 


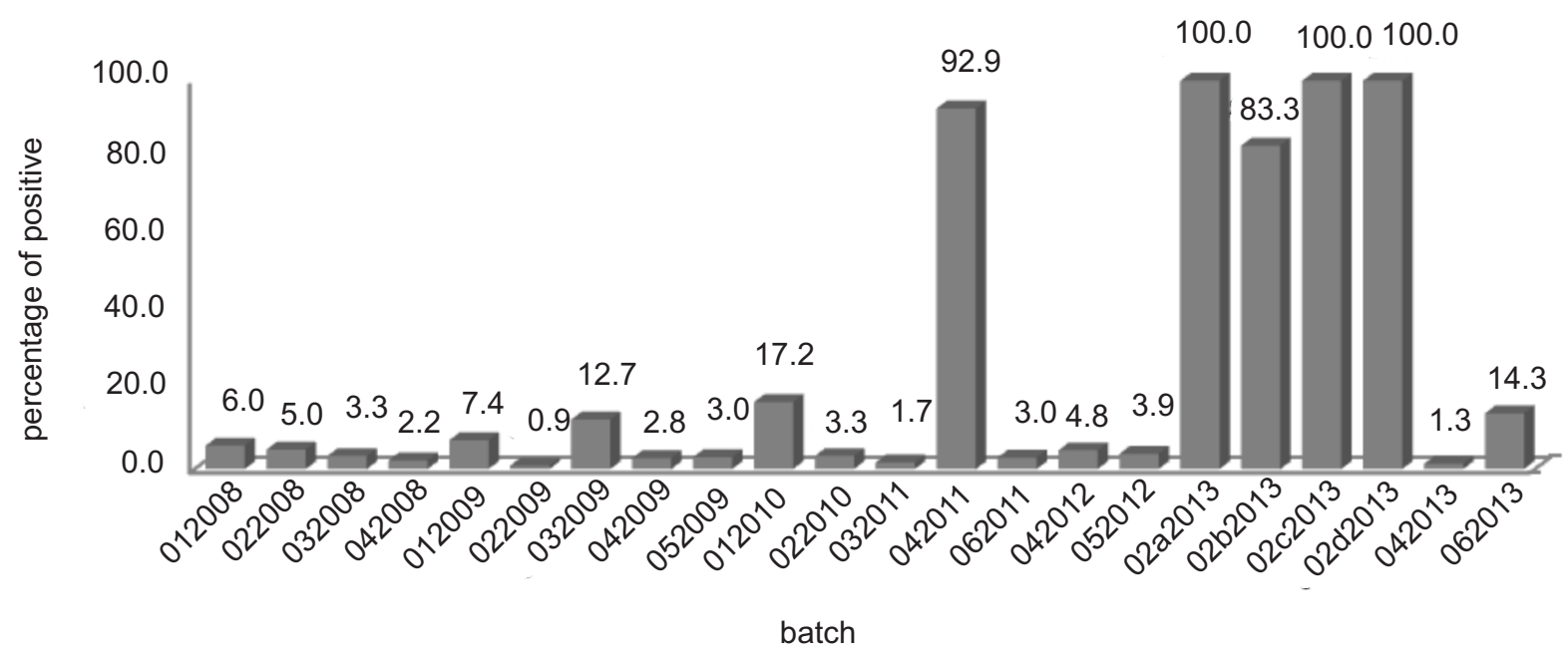

Fig. 1a. Serological assessment for RHDV antibodies in rabbits from smallholder breeding farms: total percentage of positive results.

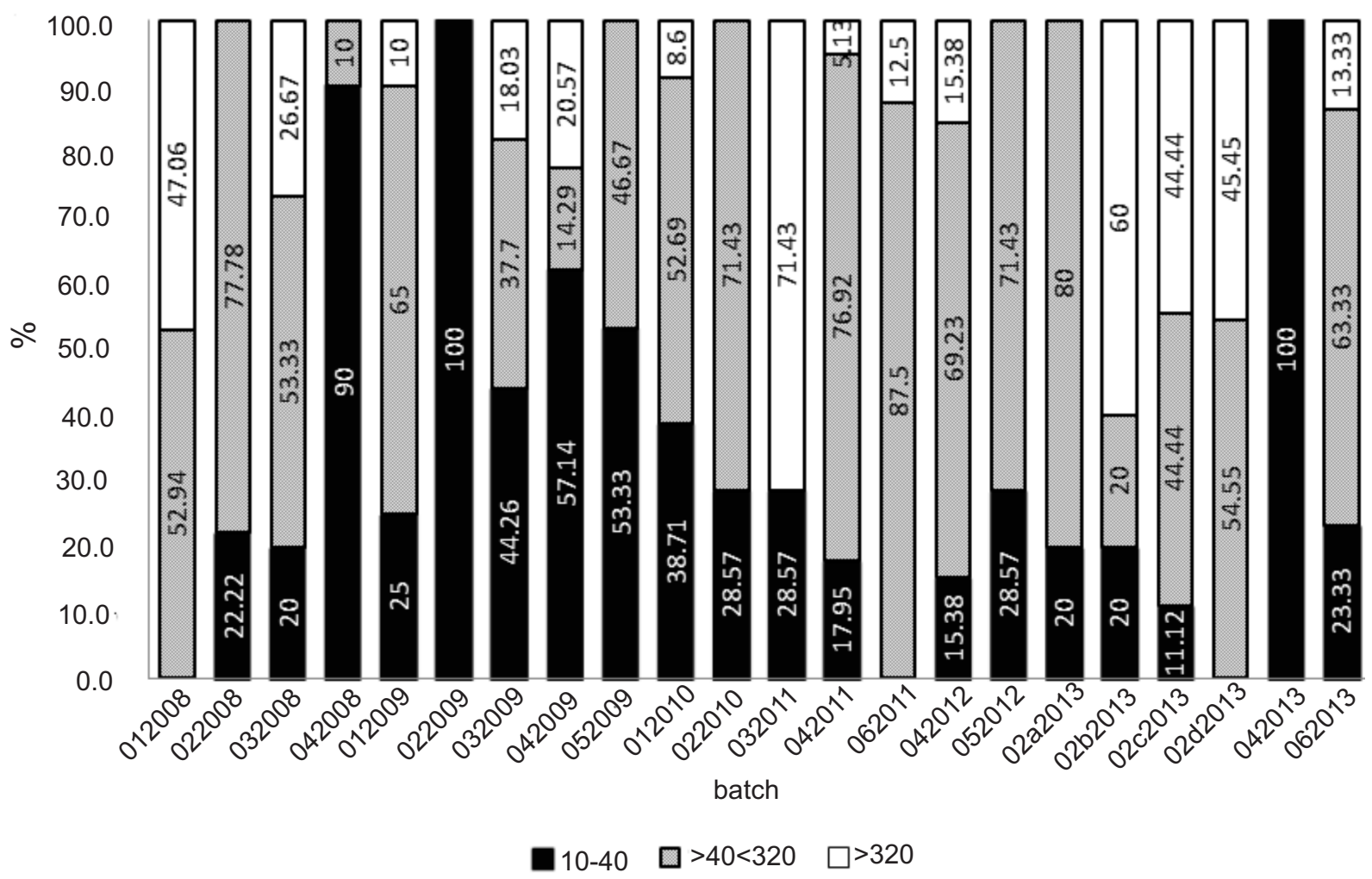

Fig. 1b. Distribution in percentage of samples of low $(10-40)$, medium $(>40<320)$ and high positive $(>320)$ titre in rabbits from smallholder breeding farms.

Fig. 1a,b and Fig. 2a,b. In these studies the presence and titer of antibodies against RHDV was detected, although it was not assessed whether vaccination induced effective immune response against RHDV infection. Antibody titers established by cELISA kit were equal or slightly higher than the titers recorded by LPBE, but generally remained within a twofold range. The serological status of 400 sera that were positive and $10 \%$ of the samples negative in ELISA (all of which represented the tested batches) was also confirmed by HI assay. In the sera from healthy rabbits from small-scale farms specific RHDV antibodies were detected in all tested batches. However, almost $94 \%$ of tested sera were negative for RHDV antibody. Positive results ranging from 1 to $17 \%$ were found in 12 batches (Fig. 1a). Among these, 31.5\% showed low $(10-40), 54.3 \%$ medium $(>40 \leq 320)$ and $14.3 \%$ (66 samples) high antibody titers of $>320$. 


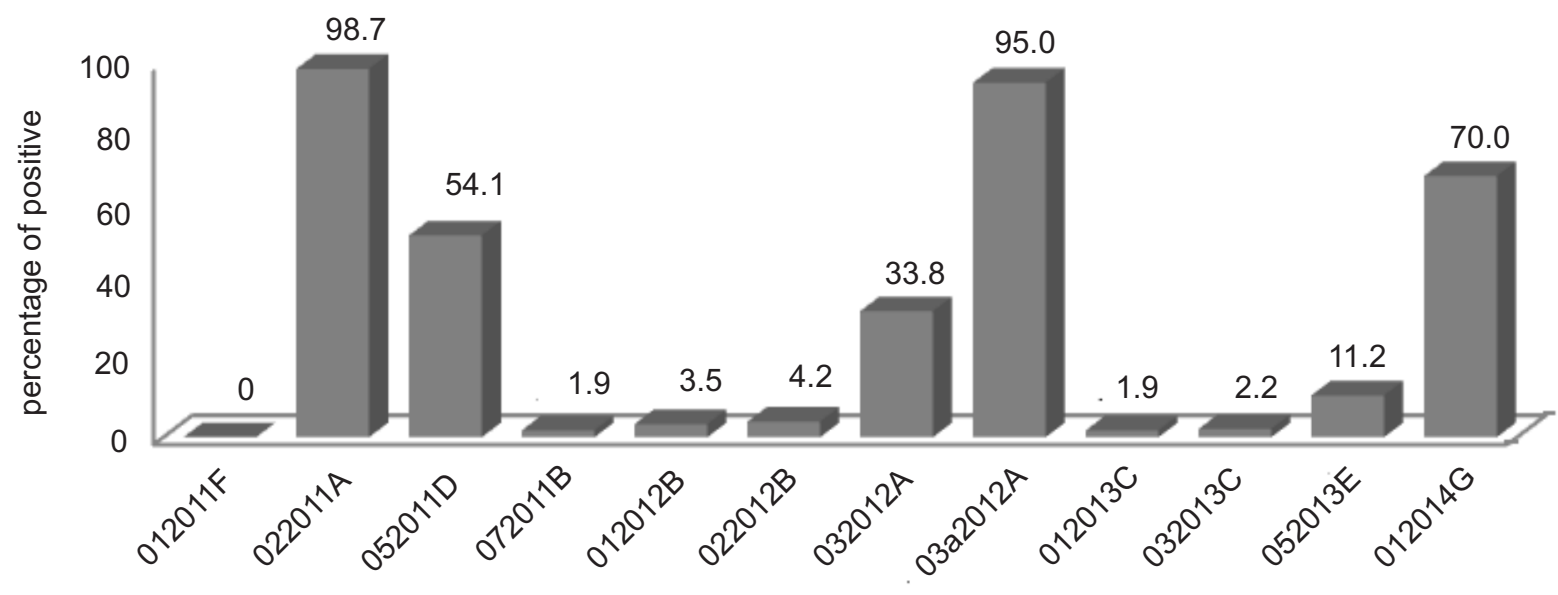

batch

Fig. 2a. Serological assessment for RHDV antibodies in rabbits from industrial farms: total percentage of positive results.

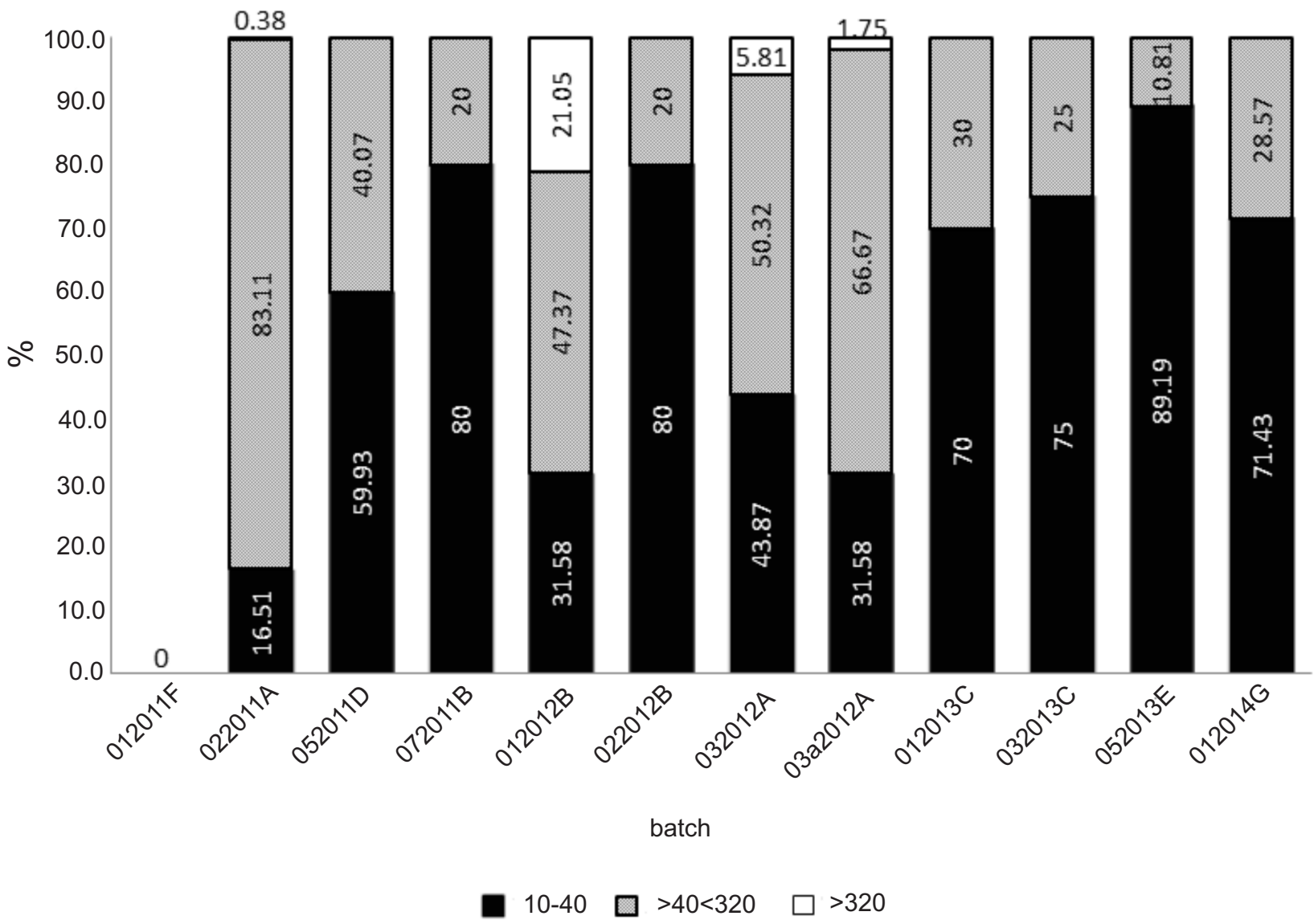

Fig. 2b. Distribution in percentage of samples of low $(10-40)$, medium $(>40<320)$ and high positive $(>320)$ titre in rabbits from industrial farms.

A higher percentage of positives was found in 5 other batches (042011, 02a-02d from 2013). In NZW rabbits (batch 042011), where both adult and young animals were regularly immunized with a bivalent vaccine against RHD and myxomatosis, 93\% seroreagents were found. The distribution of antibody levels shows that „medium titer sera” dominated over „low titer” samples. Titers exceeding a dilution of $1 / 320$ were identified in two rabbits. An equally high percentage of RHDV seroreagents and similar antibody levels were also found in the sera of mixed-breed rabbits sampled 3.5 months after RHD epizootics on 4 small 
Table 2. Seroconversion of selected test sera in HI assay using RHDV and RHDVa antigens.

\begin{tabular}{|c|c|c|c|c|c|c|}
\hline \multirow{3}{*}{ Batch } & \multirow{3}{*}{ Serum ID } & \multirow{3}{*}{ Origin } & \multicolumn{4}{|c|}{ Antibody titre } \\
\hline & & & \multicolumn{2}{|c|}{$\mathrm{HI}$} & \multirow{2}{*}{ LPB ELISA } & \multirow{2}{*}{ cELISA } \\
\hline & & & RHDV & RHDVa & & \\
\hline \multirow{8}{*}{$\begin{array}{c}02 \mathrm{a} 2013 \\
\text { (postoutbreak) }\end{array}$} & 1 & unvac. offspring $-5 \mathrm{M}$ & 320 & 320 & $80 / 160$ & 160 \\
\hline & 2 & unvac. offspring $-5 \mathrm{M}$ & 320 & 320 & $40 / 80$ & $80 / 160$ \\
\hline & 3 & unvac. offspring $-5 \mathrm{M}$ & 80 & 320 & 20 & $40 / 80$ \\
\hline & 4 & unvac. offspring $-5 \mathrm{M}$ & 80 & 1280 & 80 & 160 \\
\hline & 5 & unvac. offspring $-5 \mathrm{M}$ & 160 & 640 & 80 & $160 / 320$ \\
\hline & 6 & unvac. offspring $-5 \mathrm{M}$ & 160 & 640 & 160 & 320 \\
\hline & 7 & unvac. offspring $-5 \mathrm{M}$ & 160 & 640 & $40 / 80$ & 80 \\
\hline & 8 & unvac. offspring $-5 \mathrm{M}$ & 80 & 320 & 20 & 40 \\
\hline \multirow{2}{*}{$\begin{array}{c}02 \mathrm{~b} 2013 \\
\text { (postoutbreak) }\end{array}$} & 1 & unvac. offspring $-6 \mathrm{~W}$ & 10 & 40 & 10 & 10 \\
\hline & 2 & unvac. offspring $-6 \mathrm{~W}$ & 20 & 80 & 10 & $10 / 20$ \\
\hline \multirow{4}{*}{$\begin{array}{c}02 \mathrm{c} 2013 \\
\text { (postoutbreak) }\end{array}$} & 1 & vacc. offspring $-3 \mathrm{M}$ & 20 & 10 & $20 / 40$ & $20 / 40$ \\
\hline & 2 & vacc. offspring $-3 \mathrm{M}$ & 80 & 20 & 80 & 80 \\
\hline & 3 & vacc. offspring $-3 \mathrm{M}$ & 160 & 40 & 80 & $40 / 80$ \\
\hline & 4 & vacc. offspring $-3 \mathrm{M}$ & 40 & 80 & $40 / 80$ & $20 / 40$ \\
\hline \multirow{9}{*}{$\begin{array}{c}02 \mathrm{~d} 2013 \\
\text { (postoutbreak) }\end{array}$} & 3 & doe's 1 vacc. offspring $-3 \mathrm{M}$ & 160 & 1280 & $80 / 160$ & $160 / 320$ \\
\hline & 4 & doe's 1 vacc. offspring - $3 \mathrm{M}$ & 320 & 1280 & $80 / 160$ & $160 / 320$ \\
\hline & 5 & doe's 1 vacc. offspring - 3M & 160 & 1280 & 160 & $160 / 320$ \\
\hline & 6 & doe's 1 vacc. offspring $-3 \mathrm{M}$ & 320 & 2560 & $160 / 320$ & 320 \\
\hline & 7 & doe's 2 unvac. offspring $-6 \mathrm{~W}$ & 320 & 1280 & 160 & $160 / 320$ \\
\hline & 8 & doe's 2 unvac. offspring $-6 \mathrm{~W}$ & 640 & 10240 & $320 / 640$ & $320 / 640$ \\
\hline & 9 & doe's 2 unvac. offspring $-6 \mathrm{~W}$ & 2560 & 10240 & 1280 & $1280 / 2560$ \\
\hline & 10 & doe's 2 unvac. offspring $-6 \mathrm{~W}$ & 640 & 5120 & $640 / 1280$ & 1280 \\
\hline & 11 & doe's 2 unvac. offspring $-6 \mathrm{~W}$ & 320 & 2560 & $320 / 640$ & $320 / 640$ \\
\hline
\end{tabular}

Table 3. Seroconversion of archival sera in HI assay using RHDV and RHDVa antigens.

\begin{tabular}{|c|c|c|c|c|c|c|}
\hline \multirow{3}{*}{ Batch } & \multirow{3}{*}{ Serum ID } & \multirow{3}{*}{ Origin } & \multicolumn{4}{|c|}{ Antibody titre } \\
\hline & & & \multicolumn{2}{|c|}{$\mathrm{HI}$} & \multirow{2}{*}{ LPB ELISA } & \multirow{2}{*}{ cELISA } \\
\hline & & & RHDV & RHDVa & & \\
\hline \multirow{3}{*}{$\begin{array}{c}1994 \\
\text { (postoutbreak) }\end{array}$} & 2 & \multirow{3}{*}{$\begin{array}{l}3 \text { M survivng NZW rabbits, } \\
\text { (18 D post infection) }\end{array}$} & $640 / 1280$ & 80 & 1280 & $2560 / 5120$ \\
\hline & 4 & & $320 / 640$ & 20 & 1280 & 1280 \\
\hline & 13 & & 640 & 160 & 1280 & 1280 \\
\hline \multirow{3}{*}{$\begin{array}{c}2004 \\
\text { (postoutbreak) }\end{array}$} & 1 & \multirow{3}{*}{$\begin{array}{l}7 \text { M surviving Flemish Giant rabbits, } \\
\text { (10 D post infection) }\end{array}$} & 640 & 160 & $320 / 640$ & $640 / 1280$ \\
\hline & 3 & & 2560 & $80 / 160$ & $1280 / 2560$ & $2560 / 5120$ \\
\hline & 10 & & 160 & 40 & 80 & $80 / 160$ \\
\hline \multirow{6}{*}{$\begin{array}{l}2009 \text { (exp. vaccines) } \\
2 \text { weeks p.v. }\end{array}$} & 1 & \multirow{2}{*}{ vacc. RHDV HA-neg. antigen } & 640 & 80 & 320 & 1280 \\
\hline & 17 & & 160 & 10 & 80 & $160 / 320$ \\
\hline & 20 & \multirow{2}{*}{ vacc. RHDVa antigen I } & 40 & 640 & 40 & 320 \\
\hline & 21 & & 20 & 160 & 20 & 40 \\
\hline & 24 & \multirow{2}{*}{ vacc. RHDVa antigen II } & 320 & 1280 & $160 / 320$ & 640 \\
\hline & 25 & & 320 & 640 & 80 & 160 \\
\hline \multirow{6}{*}{$\begin{array}{l}2009 \text { (exp. vaccines) } \\
\quad \text { (10 days p.i.) }\end{array}$} & 1 & vaccinated RHDV HA-neg., infected & 5120 & 640 & $>2560$ & $>2560$ \\
\hline & 17 & RHDV & 10240 & 320 & $\geq 2560$ & $>2560$ \\
\hline & 20 & \multirow{2}{*}{ vaccinated RHDVa, infected RHDVa } & 1280 & 20480 & 1280 & 2560 \\
\hline & 21 & & 1280 & 10240 & 1280 & 1280 \\
\hline & 24 & \multirow{2}{*}{ vaccinated RHDVa, infected RHDVa } & 10240 & 20480 & $>2560$ & $>2560$ \\
\hline & 25 & & 2560 & 10240 & 1280 & $1280 / 2560$ \\
\hline
\end{tabular}


farms. $100 \%, 83 \%, 100 \%$ and $100 \%$ of both adult and young rabbits from batches $02 \mathrm{a}, 2 \mathrm{~b}, 2 \mathrm{c}$ i 2d of 2013 were RHDV positive, having titers characterized by medium and high antibody levels (Fig. 1b). The results of examination of 1- to 3-year-old females which had been vaccinated several times, and had survived an RHD epizootic, showed a slightly higher RHDV antibody titer in LPBE and cELISA than that recorded in their offspring, having titers from $1 / 80$ up to $1 / 2560$. In meat rabbits reared on industrial farms, the overall percentage of RHD sero-reactors differed to a much greater extent between the individual units. In this group, the vast majority were sera with low and medium titers (Fig. 2a, 2b). The lowest proportion $(1.85 \%-4.17 \%)$ of rabbits with detectable RHDV antibodies was found in farms $\mathrm{B}$ and $\mathrm{C}$, which were tested three and two times, respectively. A greater proportion of positive results $(11 \%)$ was found in unvaccinated, 90-day-old fattening Termond white rabbits from farm E. The highest proportion of positive results $(98.7 \%, 34 \%$ and $54 \%)$ was observed in young unvaccinated 85-day old rabbits from farms $\mathrm{A}$ and $\mathrm{D}$ and in adult vaccinated does from farms $\mathrm{A}$ and G $(95 \%, 70 \%)$.

Archival reference sera. The survey results of archival sera collected in 2004 on the industrial farm (E), from 160 RHD vaccinated adult NZW rabbit females showed the presence of RHDV antibodies in all animals. In four groups tested 3, 6, 9 and 12 months after immunization solely with monovalent vaccine, specific RHDV antibodies were detected by LPBE and $\mathrm{HI}$ tests, with titers of $1 / 10$ to $1 / 640$ and occasionally $1 / 1280$. Of the 40 vaccinated rabbits (10 of each group) no animal showed any RHD signs after challenge with classical RHDV at a $100 \mathrm{LD}_{50}$ dose. Of these, 37 rabbits showed an increase in RHD antibodies with maximum titres 1280-2560 two weeks after challenge. 25 rabbits responded with an increase in specific antibody titer of 2 to 4 times $(62.5 \%)$, six $(15 \%)$ of 6 to 12 times, and the other six had antibody titers on the same level. Three rabbits showed a twofold decline in RHD antibody titers.

HI cross-reactivity. Out of 400 sera positive in LPBE and cELISA, all were also considered positive in HI using both RHDV and RHDVa antigens. In the majority of tested sera the antibody levels examined by ELISAs and HI were comparable. However, some sera from the rabbits from smallholder farms showed differences in $\mathrm{HI}$ antibody titers depending on RHD antigen used for the assay. This type of cross-reaction which resulted in a 2-16-fold rise in antibody titers was clearly evidenced in the postoutbreak sera. The increase in reactivity with RHDVa subtype antigen was particularly evident in unvaccinated offspring aged 1.5 - 5 months (series 02a2013, 02b2013, 02d2013)
(Table 2). Conversely, in the case of several samples a reduction of HI reactivity with a RHDVa antigen and a rise of titers against classical RHDV (series 012008, 032009) was also observed. Prevalence of HI seroreactors with a differentiated profile of cross-reactivity was also revealed in control, archival sera obtained from rabbits immunized with experimental vaccines containing a specific type of RHDV or RHDVa antigen, and in postoutbreak sera collected from the survivors of 1994 and 2004 RHD epizootics (Table 3). The sera from rabbits vaccinated solely with RHDVa and infected with RHDVa produced clearly 8- to 16-fold greater titers in HI assay with homologous antigen. A similar pattern of results was obtained for the sera from animals vaccinated or vaccinated and infected with classical RHDV. Equally similar responses were obtained for postoutbreak sera collected from rabbits which survived 10-18 days in two different epizootics of RHD, caused by two classical RHDV strains, one of which is a phenotype HA-negative variant.

Virology results. Out of 247 liver samples collected at slaughter from domestic rabbits reared in small farms as well as in industrial farms all were negative in ELISA and HA assays.

\section{Discussion}

RHD is still a major disease of rabbits which threatens rabbit husbandry worldwide. The experience of many years shows that vaccination in conjuction with a prophylactic regime efficiently reduces RHDV spread in the environment. However, the relatively brief history of RHD demonstrates that periodically, due to changes in its properties, the virus obtains an advantage in this struggle. In the past, an increased incidence of RHD was always connected with a rapid spread to many other countries, across the continental boundaries (Morisse et al. 1991, Abrantes et. al 2012). Each situation of this kind was associated with the emergence of a highly pathogenic and pandemic virus subtype. The first time involved an antigenic variant of RHDVa, and more recently it has been RHDV2 with even more strongly expressed genetic variation and new biological characteristics (Le Gall-Reculé et al. 2013). Although far-reaching modifications of RHDV may be of benefit in the fight against the plague of wild rabbits in the Antipodes, for most countries the emergence of a new pathogenic form poses a direct threat to domestic rabbit production as well as to free-living wild rabbits. It is also a scientific challenge, which raises the question of the efficacy of existing vaccines.

In Poland there are no wild rabbits living freely. 
The national population of domestic rabbits is dominated by mixed-breed rabbits from small-scale farms. The open or semi-open, extensive farming system in this type of operation enables unlimited animal-enviroment contact, and poses a high risk of bacterial and viral infection introduction. In the traditional type of breeding, spreading of the virus is favored by inadequate sanitary conditions, frequent exchange of animals in close proximity, and feeding fresh green forage. From the epidemiological point of view, an important element of risk is the seasonal increase in the number of young, susceptible rabbits, from early spring to late summer. By contrast, vaccinations against RHD, if used typically, include all rabbits over the age of 6-8 weeks, ensuring the protection of an entire rabbitry.

The analysis of RHD serology in small farms revealed that the majority of tested rabbits did not show the specific humoral immunity against RHDV infections. These data are contrary to the recent results obtained by HI assay (Majer-Dziedzic et al. 2010). Our results revealed $94 \%$ of seronegative rabbits potentially highly susceptible to RHD infection, which indicates a serious risk of appearance of new outbreaks. On the other hand, an example of NZW rabbits (Series 042011) with very high seroprevalence (93\%) from the farm where vaccinations of all animals are routinely practised could be considered as evidence that such a vaccination scheme guarantees full protection of the whole herd. However, these results should not be treated as representative for other small farms, because this unit, with a total of 200 rabbits, due to animal feeding, preventive measures and vaccination strategy for both RHD and Myxomatosis, involving adult and young rabbits above 8 weeks of age, resembles an industrial-type farm. By contrast, in spite of such a high percentage of seronegative results and worse sanitary conditions on small farms, the frequency of occurrence of RHD epizootics in recent years is relatively low. This may indicate the limited circulation of the virus in the field, or partial elimination of pathogenic strains. This was also confirmed by the low percentage $(0.9 \%)$ of seropositive rabbits with antibody titers $>320$ reported on small farms. The changes of the RHD virus properties which resulted in the emergence of a new antigenic variant have also been noticed and confirmed in Poland (Chrobocińska and Mizak 2007, Fitzner 2009). The strains of RHDVa are the only subtype isolated over the past decade from RHD epizootics in our country. This fact reflects and confirms the hypothesis on the elimination of classical RHD virus from the environment (Abrantes et al. 2012).

A slightly different RHDV seroconversion was found on commercial farms (Fig. 2a). The common feature of examined rabbits was relatively equal age of the animals during sera sampling (71, 85, 90 days) and well-known immunization status for RHD due to the nonexistent immunization of fattening rabbits and vaccination at least once of the does. The results of studies in rabbits from commercial farms can be divided into those with a high number of seroreagents - in the range of several tens percent, and the low percentage of positives, from 2 to $4 \%$. The smallest rates of RHD seropositive young rabbits was found several times in the sera collected at farm B, where only single vaccination of the does was practiced, and quite unexpectedly among the ones collected from the youngest rabbits at farm $\mathrm{C}$, where two immunizations of breeding females had been reported. The highest prevalence of RHD seropositive rabbits (34\% - 98.5\%) was found in 4 batches collected at farms A and D. Among these, three series of sera were collected from 85-day-old, unvaccinated fattening rabbits and one from 60 vaccinated does, aged about 18 months. The result of antibody titre distribution in unvaccinated fattening rabbits from industrial farms indicate the presence of low and medium levels of passive immunity related to maternal antibodies (Fig. 2b). In positive sera, titres above 320 were found sporadically, and their maximum values were not higher than 1/640. A high diversity of seroconversion rate in young rabbits from commercial farms could depend on many interrelated factors, such as the quality and type of vaccines (mono versus bivalent) used for immunization, the timing and total number of vaccinations of breeders, the time elapsed since the last immunization of the females to the parturition, the intensity of their reproduction, as well as the age of young rabbits at the sampling. It can therefore be assumed that the variation of seroprevalence rate among young unvaccinated fattening rabbits coming from 6 commercial farms tested was related to their individual response, but also resulted closely from the implemented prevention program. At the same time, it is difficult to determine whether RHDV antibodies with such titres could effectively protect the animals against infection.

The present results have shown the high level of seroconversion in rabbits prophylactically vaccinated against RHDV independently of the origin of samples, i.e. type of farms (batches 042011, 03a2012, 012014). The titre of RHDV antibody found by both ELISAs and HI test is consistent with the values specified in the OIE Terrestrial Manual (Anon 2010) and our earlier results of archival sera collected from vaccinated rabbits, assessed 3, 6, 9 and 12 months from immunization. Well-documented studies of RHD seroconversion which involved domestic rabbits from industrial farms in Italy using cELISA indicated high 
levels of RHDV specific antibodies in vaccinated animals. The higher RHD serological response was recorded among the does vaccinated several times, but all young unvaccinated rabits tested at 28 days of age had passive antibodies of maternal origin. It was also demonstrated that the bivalent vaccine was as effective as a monovalent RHD vaccine (Lavazza et al. 2004). In turn, serological response against RHD in populations of wild adult rabbits in Spain previously vaccinated against RHD demonstrated seroprevalence results that ranged within $50-78 \%$. At the same time it has been shown that vaccination campaigns, introduced in order to protect the wild rabbit population of the Iberian Peninsula and to keep the ecosystem in balance, led to a reduction in their mortality due to RHD (Cooke 2002, Calvete et al. 2004, Arenas et al. 2012). The HI titers of RHDV antibody in the majority of the tested sera were consistent with both ELISAs. However, despite the close antigenic homology and genetic relationship between classic RHDV and RHDVa the results showed differences in HI reactivity in some of tested serum samples. Of a pool of positive sera collected from rabbits on the small farms, several specimens demonstrated differences in HI titers depending on RHDV antigen used. These data differ from the results of Berninger and House (1995), who studied immune responses of rabbits immunised with four diffrerent RHDV antigens isolated in Europe, Asia and North America (each should be regarded as classic RHDV), demonstrating a highly uniform cross-reactivy in HI assay. Among the offspring (1.5-5 months old) of vaccinated does which survived RHD infection 3.5 months earlier, some animals showed several times higher level of seroconversion assessed by HI using RHDVa antigen compared to reaction with classical RHDV antigen (Table 2). This was particularly clearly evident in the case of young unvaccinated rabbits (series 02d2013), where a several-fold increase in antibody titer examined by HI assay with RHDVa antigen was observed. Similar differences of reactivity in the HI test, pointing, however, to a classical antigen as the origin of infection, were confirmed by testing archival postoutbreak sera from 1994 and 2004 (Table 3).

On the basis of the obtained serological results it may be assumed that the risk of RHD on industrial farms is lower than on smallholder farms, although it cannot be excluded. The essential issue is an inadequate immune protection of the young unvaccinated rabbits, appearing when they are 7 to 8 weeks old and lasting until the end of fattening. A decline of maternal antibodies in wild rabbits between 5-11 weeks of life and susceptibility of young rabbits with high titers of pre-existing maternal RHDV antibodies to RHDV infection has been shown (Cooke et al. 2002).
The others demonstrated susceptibility to infection with non-pathogenic RCV of young domestic rabbits despite 33\% RHD seroconversion at weaning (Capucci et al. 1997). At present, this threat seems even greater because the new RHDV2 subtype (also described as RHDVb) has broken a well-known limit of insusceptibility to infection of young rabbits and caused outbreaks with fatal cases in four-week-old rabbits (Le Gall-Recule et al. 2013). Although it was not confirmed in experimental studies with rabbits, it can be assumed that the vaccination of does from the basic herd may affect the protective potential of the young unvaccinated rabbits in this age group. On the basis of the level of antibody titers it can be assumed that a significant proportion of seropositive results found in test samples are related to the commercial vaccines used. It is known that inactivated vaccines containing classic RHDV antigen are efficient in protection against infection with both RHDV and RHDVa subtypes, but due to the large genetic and antigenic changes they could not be so against RHDV2 (Le Gall-Recule et al. 2013).

In conclusion, it can be stated that regardless of the type of farming, the native population of domestic rabbits in the vast majority is free from antibodies against RHDV and can be susceptible to RHDV infection. The epidemiological situation on industrial farms can be well-controlled, which results from the ongoing prevention program, and involves 2 or 3 fold vaccination of does and relatively short period of breeding rabbits during the fattening period (approx. 80 days). However, in the case of smaller farms the risk of infection is significantly higher, and although the economic losses in such farms are not so severe on the macro scale and are limited to the individual farms, from the epizootic point of view, these smaller farms present a much greater danger of new outbreaks and can be the source of epidemics.

\section{References}

Abrantes J, van der Loo W, Le Pendu J, Esteves PJ (2012) Rabbit haemorrhagic disease (RHD) and rabbit haemorrhagic disease virus (RHDV): a review. Vet Res 43: 12.

Anon (2010) World Organisation for Animal Health. Chapter 2.6.2 Rabbit haemorrhagic disease (Version adopted in May 2010). In: Manual of Diagnostic Tests and Vaccines for Terrestrial Animals, 7th ed., OIE, Paris.

Arenas AJ, Napp SB, Arenas-Montes A, Borge C, Carbonero A, Perea A, Cadenas R, Garcia-Bocanegra I (2012) Serological response against myxoma virus and rabbit hemorrhagic disease virus in European wild rabbits using commercial vaccines. J Wildl Manag 76: 102-107.

Berninger ML, House C (1995) Serologic comparison of four isolates of rabbit hemorrhagic disease virus. Vet Microbiol 47: 157-165. 
Bruce JS, Twigg LE (2004) Rabbit haemorrhagic disease virus: serological evidence of a non-virulent RHDV-like virus in south-western Australia. Wildl Res 31: 605-612.

Calvete C, Estrada R, Lucientes J, Osacar JJ, Villafuerte $R$ (2004) Effects of vaccination against viral haemorrhagic disease and myxomatosis on long-term mortality rates of European wild rabbits. Vet Rec 155: 388-392.

Cancellotti FM, Renzi M (1991) Epidemiology and current situation of viral haemorrhagic disease of rabbits and the European brown hare syndrome in Italy. Rev Sci Tech 10: 409 - 422.

Capucci L, Fusi P, Lavazza A, Pacciarini ML, Rossi C (1996) Detection and preliminary characterization of a new rabbit calicivirus related to rabbit hemorrhagic disease virus but nonpathogenic. J Virol 70: 8614-8623.

Capucci L, Nardin A, Lavazza A (1997) Seroconversion in an industrial unit of rabbits infected with a non-pathogenic rabbit haemorrhagic disease-like virus. Vet Rec 140: 647-650

Capucci L, Fallacara F, Grazioli S, Lavazza A, Pacciarini ML, Brocchi E (1998) A further step in the evolution of rabbit hemorrhagic disease virus: the appearance of the first consistent antigenic variant. Virus Res 58: 115-126.

Chrobocińska M, Mizak B (2007) Phylogenetic analysis of partial capsid protein gene of rabbit haemorrhagic disease virus (RHDV) strains isolated between 1993 and 2005 in Poland. Bull Vet Inst Pulawy 51: 189-197.

Cooke BD, Robinson AJ, Merchant JC, Nardin A, Capucci L (2000) Use of ELISAs in field studies of rabbit haemorrhagic disease (RHD) in Australia. Epidemiol Infect 124: 563-576.

Cooke BD (2002) Rabbit haemorrhagic disease: field epidemiology and the management of wild rabbit populations. Rev Sci Tech 21: 347-358.

Delibes-Mateos M, Ferreira C, Carro F, Escudero MA, Gortazar C (2014) Ecosystem effects of variant rabbit hemorrhagic disease virus, Iberian Peninsula. Emerg Infect Dis 20: 2166-2168.

Fitzner A (2009) Characterisation and immunogenic properties of Polish strains of RHD virus. Bull Vet Inst Pulawy 53: $575-582$.
Fitzner A, Niedbalski W (1996) Application of the ELISA for the virologic and serologic diagnosis of viral haemorrhagic disease of rabbits (VHD). Bull Vet Inst Pulawy 40: 69-76.

Górski J, Mizak B, Mizak Z, Komorowski A (1988) Clinical and anatomopathological features of rabbits peste (Viral haemorrhagic disease of rabbits). Życie Wet 63: 266-269.

Lavazza A, Graziani M, Tranquillo VM, Botti G, Palotta C, Cerioli M, Capucci L (2004) Serological evaluation of the immunity induced in commercial rabbits by vaccination for myxomatosis and RHD. Procedings of $8^{\text {th }}$ World Rabbits Congress, September 7-10, 2004, Puebla Mexico, pp 569-575.

Le Gall G, Arnauld C, Boilletot E, Morisse JP, Rasschaert D (1998) Molecular epidemiology of rabbit haemorrhagic disease virus outbreaks in France during 1988 to 1995. J Gen Virol 79: 11-16.

Le Gall-Recule G, Lavazza A, Marchandeau S, Bertagnoli S, Zwilgenstein F, Cavadini P, Martinelli N, Lombardi G, Guerin JL, Lemaitre E, Decors A, Boucher S, Le Normand B, Capucci L (2013) Emergence of a new lagovirus related to Rabbit Haemorrhagic Disease Virus. Vet Res 44: 81.

Liu SJ, Xue HP, PU BQ, Qian NH (1984) A new viral disease in rabbits. Anim Hus Vet Med 16: 253-255.

Majer-Dziedzic B, Szkucik K (2010) Serological evaluation of antibody titres to rabbit haemorrhagic disease. Med Weter 66: 206-209.

Marchandeau S, Chantal J, Portejoie Y, Barraud S, Chaval Y (1998) Impact of viral hemorrhagic disease on a wild population of European rabbits in France. J Wildl Dis 34: 429-435.

Morisse JP, Le Gall G, Boilletot E (1991) Hepatitis of viral origin in Leporidae: introduction and aetiological hypotheses. Rev Sci Tech 10: 283-295.

Ohlinger VF, Haas B, Meyers G, Weiland F, Thiel HJ (1990) Identification and characterization of the virus causing rabbit hemorrhagic disease. J Virol 64: 3331-3336. 